\title{
Evaluating carbapenem restriction practices at a private hospital in Manila, Philippines as a strategy for antimicrobial stewardship
}

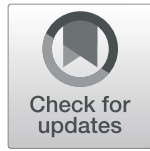

Kaitlin F. Mitchell ${ }^{1,5}$, Nasia Safdar ${ }^{1,2,3}$ and Cybele L. Abad ${ }^{4^{*}}$ (D)

\begin{abstract}
Background: Hospital antimicrobial stewardship programs are especially critical in countries such as the Philippines, where antibiotic resistant infections are highly prevalent. At the study institution in Manila, Philippines, a Prior Approval for Restricted Antimicrobials (PARA) is required for non-infectious disease specialists to prescribe certain antimicrobials, including carbapenems. PARA request forms include specification of empiric or definitive therapy based on diagnostic tests. Recommended duration of therapy is typically 3 days for empiric use and 7 days for definitive, with possible extension upon specialist approval.

Methods: The study took place at an 800-bed tertiary hospital. We performed a retrospective review of patient medical records and laboratory reports dating from January 1 to December 31, 2016. Information related to patient demographics, carbapenem prescription, laboratory diagnosis, and therapy were compiled. Carbapenem prescriptions were classified as 'adherent' or 'non-adherent' according to clinical guidelines related to infection diagnosis, treatment duration, and de-escalation.

Results: Of the 185 patients on carbapenem therapy, Prescriptions of carbapenems were either definitive $(n=56)$, empiric $(n=127)$, or prophylactic $(n=2)$ as defined by the ordering provider. 69 out of $185(37 \%)$ prescriptions were deemed non-adherent to guidelines, despite receiving approvals for their respective requests. Of these, $72 \%$ were nonadherent due to failure to de-escalate the carbapenem and $28 \%$ were non-adherent due to an incomplete course of therapy.

Conclusion: Despite initial PARA approval for carbapenem therapy, 37\% of prescriptions were non-guideline-adherent, highlighting the ongoing challenges in implementing this type of stewardship strategy. In order to increase the effectiveness of PARA, additional approaches may be warranted, including the application of strict policies which reinforce follow-up of available culture results, justification of therapy extension, or referral to an infectious disease specialist.
\end{abstract}

Keywords: Antimicrobial stewardship, Philippines, Carbapenem, Empiric and definitive prescriptions

\section{Background}

Antibiotic resistance is disproportionately abundant in low- and middle- income countries such as the Philippines. Multiple factors contribute to this: patients can often obtain antibiotics without a prescription, many healthcare providers write unnecessary prescriptions, and infectious disease surveillance systems may be inadequate [1-3]. Studies in the Philippines have identified

\footnotetext{
* Correspondence: cybelemd@yahoo.com

${ }^{4}$ Department of Medicine, Section of Infectious Diseases, The Medical City Hospital, Ortigas Ave, Pasig City, Philippines

Full list of author information is available at the end of the article
}

issues such as self-prescription, use of antibiotics for unconfirmed bacterial infections, and dispensing of incomplete antibiotic courses by both community pharmacies and clinics $[4,5]$. Given these factors, patients are more likely to develop antibiotic resistance to these readilyavailable drugs and require more advanced antibiotics. Healthcare facilities are the source of such antibiotics for many individuals, and have the ability to provide drugs of 'last resort' that are not typically available elsewhere [3]. Inappropriate use of these antimicrobial agents is a significant contributor to the development of multi-drug

(C) The Author(s). 2019 Open Access This article is distributed under the terms of the Creative Commons Attribution 4.0 International License (http://creativecommons.org/licenses/by/4.0/), which permits unrestricted use, distribution, and reproduction in any medium, provided you give appropriate credit to the original author(s) and the source, provide a link to the Creative Commons license, and indicate if changes were made. The Creative Commons Public Domain Dedication waiver (http://creativecommons.org/publicdomain/zero/1.0/) applies to the data made available in this article, unless otherwise stated. 
resistant organisms (MDROs) and untreatable infections [6].

Similar to other healthcare facilities in the region, our hospital has high rates of MDROs. For example, data collected from 2016 revealed high rates of Pseudomonas aeruginosa with resistance to last-resort antibiotics: among respiratory isolates from the intensive care unit, 31 and $37 \%$ were resistant to piperacillin-tazobactam and carbapenems, respectively (unpublished data).

To help preserve the effectiveness of existing antimicrobials, many hospitals have implemented antimicrobial stewardship programs (ASPs). The primary goal of any ASP is to optimize clinical outcomes while minimizing the unintended consequences of antimicrobial utilization, which include the development of resistance and toxicity $[7,8]$. These programs can be especially critical in reducing unnecessary carbapenem utilization $[9,10]$. One evidence-based strategy for achieving this is formulary restriction with prior authorization for select agents $[7,8]$. Formulary restriction can be accomplished by requiring prior approval for use by an infectious disease (ID) specialist. While this method can be effective, it requires highly-trained personnel [11].

The present study examines one component of the ASP at our large, tertiary hospital located in Manila, Philippines: the Prior Approval for Restricted Antimicrobials (PARA). The role of formulary restriction with prior authorization for select agents has not been evaluated at this institution. We undertook a retrospective study to evaluate the effect of this strategy on carbapenem prescribing.

\section{Methods}

\section{Study setting and population}

This retrospective study was conducted at The Medical City, a private, 800 -bed, tertiary hospital in Manila, Philippines. This hospital has had an ASP in place since 2002. The Prior Approval for Restricted Antimicrobials (PARA) is an integral part of this program that requires approval from an infectious disease (ID) specialist prior to the prescription of restricted antibiotics, including carbapenems (meropenem, ertapenem, and imipenem) (Additional file 1).

We evaluated patients with a request for carbapenem therapy through the PARA process between January 1 and December 31, 2016.

\section{Description of prior approval for restricted antimicrobials (PARA)}

PARAs can be requested by phone $24 \mathrm{~h}$ a day, 7 days a week, and are granted by a clinical pharmacist after approval by the ID specialist. Following both clinical and institutional guidelines, definitive prescriptions of 7 days are recommended for infections with a laboratoryconfirmed diagnosis while empiric prescriptions for 3 days are suggested for infections prior to diagnosis [1214]. Prophylactic prescriptions are those given for 1-2 doses, prior to device insertion or surgery (Additional file 2). Longer durations of therapy must be approved by a PARA extension, otherwise the pharmacist releases an automatic stop order. Once laboratory data is available for empiric prescriptions, the carbapenem should be deescalated if warranted, though this is not specifically indicated in the hospital's policy.

\section{Inclusion and exclusion criteria}

Inclusion criteria were either the approval or disapproval for any carbapenem prescription within the study period. For patients with multiple PARA requests, only the first approved request for carbapenem use was included. Subsequent requests were included in the analysis only if they were for a different indication (i.e. different infection site or different carbapenem requested). Patients were excluded from the study if their carbapenem was prescribed by an ID specialist, as these physicians do not need a PARA request to prescribe carbapenems. Patients were also excluded if the PARA request was for a restricted antibiotic other than a carbapenem.

\section{Data collection and analysis}

A list of patients with PARA requests was compiled by the hospital's infection control department. Patient record numbers from this list were used to manually search for medical records from the hospital's online database (Medical Information Data Analysis System, or MIDAS). Electronic laboratory records were queried to obtain culture results and antibiotic susceptibility data.

Data collected included: patient demographics, comorbidities, duration of hospital stay, antimicrobial dosage and duration of therapy, culture data (isolated pathogen, infection site, and susceptibility data), and patient outcomes (survival or death at end of hospital stay). MDROs were defined as organisms with reported resistance to one or more antibiotic classes. Carbapenem prescriptions were categorized as guideline-adherent or nonadherent, with prescription adherence defined as follows: 1) carbapenems de-escalated based on laboratory data, or 2) completion of the antibiotic course. In addition, antibiotic courses exceeding the recommended duration for empiric and definitive prescriptions were considered adherent if there was an ID specialist assigned to the patient case to approve the PARA extension.

\section{Statement of ethical compliance}

The study was approved by the Institutional Review Board (IRB) at the site in the Philippines. The study was considered exempt from review by the University of Wisconsin-Madison IRB, as it qualified as a quality improvement study. 


\section{Results}

275 patient cases were identified from the initial database of PARA requests in 2016. After applying exclusion criteria, 185 cases remained for analysis. Prescriptions of carbapenems were either definitive $(n=56)$, empiric $(n=127)$, or prophylactic $(n=2)$ as defined by the ordering provider. For the two cases with prophylactic carbapenems, prescriptions were requested during the placement of a surgical device, because of history of prior drug resistance. The median patient age was 75.5 years (range 23 to 98 ), $45 \%$ of cases were male, and common comorbidities included type 2 diabetes and hypertension. The most common site of infection was the respiratory tract (77.3\% of cases) (Table 1).

Organism identification data was collected from the hospital's microbiology laboratory reports. 276 clinical isolates were reported from the 185 patients. Hospital acquired enteric and Gram-negative organisms that are commonly associated with carbapenem resistance accounted for $56.2 \%$ of diagnoses (Table 2). 229 of 276 $(83.0 \%)$ of the isolates had susceptibility testing reported, and 101 of these (44.0\%) were MDROs.

Using the guidelines defined above, 69 out of 185 (37\%) prescriptions were deemed non-adherent, despite receiving approvals for their respective PARA requests.
Of these, 50 (72\%) were non-adherent as there was no de-escalation of the carbapenem and 19 (28\%) were due to an incomplete course of therapy. These values were also calculated within groups for empiric, definitive, and prophylactic prescriptions and are shown in Table 3.

Several notable details related to these non-adherent prescription classifications were encountered in the patient records. First, failure to de-escalate from carbapenems was sometimes observed in the absence of a laboratory result (in contrast to a result of "No organism" or "Other organism"). Second, an incomplete course of antibiotics was considered non-adherent only for reasons within the control of the provider. These reasons included 1) the discharge of a patient prior to full carbapenem course (without indication for antibiotic continuation as an outpatient), 2) patient or family member's refusal to finish the carbapenem course, sometimes due to financial concerns, 3) or note of clinical improvement/resolution with the carbapenem that justified discontinuation of therapy, or 4) no reason was stated in the charts.

\section{Discussion}

Our study describes the PARA program, the approval process for restricted antibiotics at a hospital in Manila,

Table 1 Characteristics of patients with a Prior Approval for Restricted Antimicrobials (PARA) request between January 1 and December 31, 2016. Manila, Philippines. "Evaluating carbapenem restriction practices at a private hospital in Manila, Philippines as a strategy for antimicrobial stewardship"

\begin{tabular}{|c|c|c|c|c|}
\hline & $\begin{array}{l}\text { Total } \\
\mathrm{n}(\%)^{\mathrm{a}}\end{array}$ & $\begin{array}{l}\text { Definitive } \\
\mathrm{n}(\%)\end{array}$ & $\begin{array}{l}\text { Empiric } \\
\mathrm{n}(\%)\end{array}$ & $\begin{array}{l}\text { Prophylactic } \\
\mathrm{n}(\%)\end{array}$ \\
\hline Group size $(\mathrm{N})$ & 185 & 56 & 127 & 2 \\
\hline Age (median years) & 75.5 & 78.4 & 72.7 & 75.0 \\
\hline Gender (male) & $83(45 \%)$ & $30(54 \%)$ & $52(41 \%)$ & $1(50 \%)$ \\
\hline Total \# comorbidities of interest & 268 & 83 & 183 & 2 \\
\hline Average \# comorbidities per patient & 1.45 & 1.48 & 1.44 & 1.00 \\
\hline Chronic Obstructive Pulmonary Disorder & 25 & 8 & 16 & 1 \\
\hline Hypertension & 102 & 36 & 66 & 0 \\
\hline Diabetes Mellitus & 66 & 23 & 43 & 0 \\
\hline Malignancy (any, active) & 51 & 9 & 41 & 1 \\
\hline Hemodialysis & 25 & 6 & 19 & 0 \\
\hline None & 28 & 7 & 20 & 1 \\
\hline \multicolumn{5}{|l|}{ Outcome } \\
\hline Length of hospital days (median days) & 14.0 & 14.5 & 14.0 & 15.0 \\
\hline Mortality & $42(23 \%)$ & $10(18 \%)$ & $32(25 \%)$ & $0(0 \%)$ \\
\hline Cases with same recurrent infection within 30 days of discharge & $14(8 \%)$ & $2(4 \%)$ & $12(9 \%)$ & $0(0 \%)$ \\
\hline Duration of therapy (median days) & 6.5 & 7.0 & 5.0 & 11.0 \\
\hline \multicolumn{5}{|l|}{ Site of infection for PARA request } \\
\hline Respiratory & 145 & 30 & 113 & 2 \\
\hline Blood & 18 & 8 & 10 & 0 \\
\hline Gastrointestinal & 1 & 1 & 0 & 0 \\
\hline Genitourinary & 39 & 24 & 15 & 0 \\
\hline Wound/surgical site & 2 & 1 & 1 & 0 \\
\hline
\end{tabular}

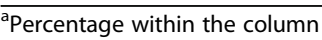


Table 2 Bacterial species identified as reported by the microbiology laboratory $(n=276)$. 2016, Manila, Philippines. "Evaluating carbapenem restriction practices at a private hospital in Manila, Philippines as a strategy for antimicrobial stewardship"

\begin{tabular}{ll}
\hline Organism & $\begin{array}{l}\text { Number of isolates, } \\
N=276 \\
\mathrm{n}(\%)\end{array}$ \\
\hline Klebsiella pneumoniae & $53(19.2)$ \\
Escherichia coli & $42(15.2)$ \\
Acinetobacter baumanii & $20(7.2)$ \\
Pseudomonas aeruginosa & $15(5.4)$ \\
Enterobacter cloacae & $13(4.7)$ \\
Citrobacter spp. $^{\text {a }}$ & $6(2.2)$ \\
Klebsiella oxytoca & $2(0.7)$ \\
Proteus mirabilis & $2(0.7)$ \\
Serratia marcescens & $2(0.7)$ \\
Negative & $36(13.0)$ \\
Other & $85(30.8)$ \\
Candida albicans & $38(44.7)^{\mathrm{b}}$ \\
Staphylococcus aureus & $15(17.6)$ \\
Stenotrophomonas maltophila & $10(11.8)$ \\
Enterococcus faecalis & $3(3.5)$ \\
Achromobacter xylosoxidans & $2(2.4)$ \\
Aeromonas hydrophila & $2(2.4)$ \\
Enterococcus faecium & $2(2.4)$ \\
Haemophilus influenzae & $2(2.4)$ \\
Singletons & $11(12.9)$ \\
\hline
\end{tabular}

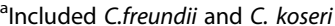

bercentage from 'Other' group, containing organisms not typically treated with carbapenems

Philippines. Since its inception in 2002, the program has not been evaluated in terms of subsequent antibiotic prescriptions following the approval process. In our study, we found the majority (63\%) of prescriptions using PARA were guideline-adherent.

It is challenging, especially in resource-limited environments, to maintain an ASP with coordination of physicians, the laboratory, infection control, and clinical pharmacists [1, 15]. At this institution, we found that carbapenem prescriptions granted within the PARA program were more often adherent to clinical practice guidelines than not. In addition, many patients who were discharged prior to the end of their prescriptions were provided instruction for outpatient care, which often involved intravenous administration. Despite these program strengths, the proportion of prescriptions that deviated from clinical guidelines (37\%) underscores some gaps of the current strategy.

The PARA program does not mandate adjustment of therapy upon the availability of microbiological culture results. We found this was particularly an issue for empiric prescriptions, which are meant to provide reasonable antibiotic therapy until culture data are available, usually within 48 to $72 \mathrm{~h}$ [16]. Even when laboratory results indicated a negative culture or an irrelevant organism, carbapenems were not always discontinued or de-escalated to a narrowspectrum antibiotic. This scenario, which occurred for $44.5 \%$ of PARA prescriptions, represents a clear point where carbapenems should be de-escalated. The decisions regarding therapy in these cases were often left to providers rather than referred to an ID specialist. Full utilization of a microbiology laboratory's testing capability is critical to the success of an ASP, as has been demonstrated in other healthcare settings with limited-resources [15, 17, 18].

Lack of clinical confidence has been cited as a barrier to successful de-escalation [19] - if an antibiotic is noticeably improving a patient's symptoms, there may be reluctance to change. Previous studies have shown that de-escalation strategies reduce antimicrobial resistance levels and patient mortality without compromising patient safety, but also acknowledge difficulties in implementation [19-21]. As our study corroborates, de-escalation policies should be supplemented by implementation support; providing physicians with additional support from ID specialists or clinical pharmacists at these key decision-making moments, perhaps through direct audit and feedback, or requiring follow up consults by phone or in-person, are essential. Asking providers to describe their prescribing choices at set time points has been shown to increase accountability [22], and could be a useful part of these

Table 3 Number of carbapenem prescriptions following Prior Approval for Restricted Antimicrobials (PARA) by adherence to clinical guidelines and by the reason for non-adherence. 2016, Manila, Philippines. "Evaluating carbapenem restriction practices at a private hospital in Manila, Philippines as a strategy for antimicrobial stewardship"

\begin{tabular}{lllll}
\hline & Total $\mathrm{n}(\%)$ & $\begin{array}{l}\text { Definitive } \\
\mathrm{n}(\%)\end{array}$ & $\begin{array}{l}\text { Empiric } \\
\mathrm{n}(\%)\end{array}$ & $\begin{array}{l}\text { Prophylactic } \\
\mathrm{n}(\%)\end{array}$ \\
\hline Total cases & 185 & 56 & 127 & 2 \\
$\quad$ Guideline-adherent & $116(63 \%)$ & $43(77 \%)$ & $71(56 \%)$ & $2(100 \%)$ \\
$\quad$ Non-guideline-adherent & $69(37 \%)$ & $13(23 \%)$ & $56(44 \%)$ & $0(0 \%)$ \\
Total non-guideline-adherent cases & 69 & 13 & 56 & - \\
$\quad$ No de-escalation & $50(72 \%)$ & $5(38 \%)$ & $45(80 \%)$ & - \\
Incomplete course & $19(28 \%)$ & $8(62 \%)$ & $11(20 \%)$ & - \\
\hline
\end{tabular}


consults. In addition, stewardship interventions led by ID specialists have led to reduced carbapenem utilization and subsequent reduction in antibiotic resistance rates $[9,10]$.

Another reason for non-adherent prescriptions was premature shortening of antibiotic courses, which is discouraged in current guidelines [23, 24]. Both the definitive and empiric prescription groups in our study included patients given incomplete courses of carbapenems. Our analysis excluded cases with therapy that was shortened for reasons beyond the control of a provider (e.g. patient death). However, other reasons for non-adherent carbapenem shortening may be preventable. Patients who need ongoing carbapenem therapy should enter a formal outpatient antibiotic treatment program. In several cases, patients' financial concerns were noted as the reason for incomplete antibiotic courses, representing an important area of future work in stewardship programs.

In our cohort, K. pneumoniae and $E$. coli were the most commonly isolated organisms at 19.2 and $15.2 \%$, respectively. This distribution of pathogens is unsurprising and mirrors national data: in a 2017 report of 76,892 clinical isolates from the national Antimicrobial Resistance Surveillance Program [25], K. pneumoniae (16.4\%) and E. coli (11.6\%) accounted for the top 2 isolated organisms as well.

Our study had several limitations. Review of patient charts involved reading numerous scanned pages with handwritten text. Since these were non-searchable for key words, certain notes of interest may have been missed. Furthermore, many patient records did not contain any laboratory reports for the infection of interest. Based on the information available in the charts at this institution, the underlying reasons for absent cultures were unclear - the test may have been ordered but not carried out because of the inability of the patient to provide a sample; in most cases of hospital-acquired pneumonia, for example, the patient is unable to expectorate sputum. We were unable to capture carbapenem prescriptions made by ID physicians which can provide useful information regarding overall carbapenem use within the hospital. Also, the use of ancillary laboratory diagnostics such as procalcitonin, which may have been used to help determine treatment length, was not captured in this study. Finally, this was a single-center analysis conducted at a private hospital. This facility has an established framework for infection control and antimicrobial stewardship, so findings for this specific program may not be applicable to facilities without an ASP currently in place.

\section{Conclusions}

The global challenge presented by antimicrobial resistance necessitates the establishment of ASPs to curtail inappropriate use of antibiotics in healthcare facilities. Our study highlights some of the issues that may be faced by institutions in resource-limited settings when implementing these policies. Opportunities for intervention include greater utilization of available laboratory resources and mandating the adjustment of therapy based on test results. Increasing decision-making support for providers on optimizing carbapenem use may also be of benefit.

\section{Additional files}

Additional file 1: Algorithm for PARA (Prior Approval for Restricted Antimicrobials). (DOCX $145 \mathrm{~kb}$ )

Additional file 2: Summary of empiric antibiotic therapy guidelines used at the study site for developing the PARA program. Guidelines were derived from national and local guidelines in the Philippines for empiric antibiotic therapy $[11,12]$ and institutional antibiogram data (data not shown). (DOCX $18 \mathrm{~kb})$

Abbreviations

ASP: Antimicrobial stewardship program; ID: Infectious Disease; MDRO: Multi Drug Resistant Organism; PARA: Prior Approval for Restricted Antimicrobials

\section{Acknowledgements \\ Not applicable.}

Previous presentation

No portion of the data or manuscript have been published or submitted for publication elsewhere.

\section{Authors' contributions}

KFM drafted and edited the manuscript, and contributed to study design, data acquisition and data analysis. NS contributed to the study design, data analysis, and critical manuscript editing. CLA contributed to the study design, data analysis, and critical manuscript editing. All authors read and approved the final version of this manuscript.

\section{Funding}

This project was funded through a Graduate Student Research Award from the University of Wisconsin-Madison Global Health Institute awarded to Kaitlin Mitchell.

\section{Availability of data and materials}

The datasets used and/or analyzed during the current study are available from the corresponding author on reasonable request.

\section{Ethics approval and consent to participate}

The study was approved by the Institutional Review Board (IRB) at The Medical City in the Philippines. The study was considered exempt from review by the University of Wisconsin-Madison IRB.

\section{Consent for publication}

Not applicable.

\section{Competing interests}

The authors declare that they have no competing interests.

\section{Author details}

${ }^{1}$ Division of Infectious Diseases, Department of Medicine, University of Wisconsin-Madison, Madison, WI, USA. ${ }^{2}$ William S. Middleton Memorial Veterans Hospital, Madison, WI, USA. ${ }^{3}$ Infection Control Department, University of Wisconsin-Madison, Madison, WI, USA. ${ }^{4}$ Department of Medicine, Section of Infectious Diseases, The Medical City Hospital, Ortigas Ave, Pasig City, Philippines. ${ }^{5}$ Present Address: Laboratory and Genomic Medicine, Department of Pathology and Immunology, Washington University School of Medicine in St. Louis, St. Louis, MO, USA. 
Received: 1 February 2019 Accepted: 13 June 2019

Published online: 04 July 2019

\section{References}

1. Allegranzi B, Pittet D. Healthcare-associated infection in developing countries: simple solutions to meet complex challenges. Infection Control \& Hospital Epidemiology. 2007;28:1323-7.

2. Safdar N, Sengupta S, Musuuza JS, Juthani-Mehta M, Drees M, Abbo LM, Milstone AM, Furuno JP, Varman M, Anderson DJ, et al. Status of the prevention of multidrug-resistant organisms in international settings: a survey of the Society for Healthcare Epidemiology of America research network. Infect Control Hosp Epidemiol. 2016:1-8.

3. The Philippine action plan to combat antimicrobial resistance: one health approach. https://arsp.com.ph/wp-content/uploads/2016/07/Action-Plan-final_ orig.pdf.

4. Lansang MA, Lucas-Aquino R, Tupasi TE, Mina VS, Salazar LS, Juban N, Limjoco TT, Nisperos LE, Kunin CM. Purchase of antibiotics without prescription in Manila, the Philippines. Inappropriate choices and doses. J Clin Epidemiol. 1990;43:61-7.

5. Hardon AP. The use of modern pharmaceuticals in a Filipino village: doctors' prescription and self medication. Soc Sci Med. 1987;25:277-92.

6. Bartlett JG. A call to arms: the imperative for antimicrobial stewardship. Clinical infectious diseases: an official publication of the Infectious Diseases Society of America. 2011;53(Suppl 1):S4-7.

7. Dellit TH, Owens RC, McGowan JE Jr, Gerding DN, Weinstein RA, Burke JP, Huskins WC, Paterson DL, Fishman NO, Carpenter CF, et al. Infectious Diseases Society of America and the Society for Healthcare Epidemiology of America guidelines for developing an institutional program to enhance antimicrobial stewardship. Clinical infectious diseases: an official publication of the Infectious Diseases Society of America. 2007:44:159-77.

8. Drew RH, White R, MacDougall C, Hermsen ED, Owens RC, Jr.: Insights from the Society of Infectious Diseases Pharmacists on antimicrobial stewardship guidelines from the Infectious Diseases Society of America and the Society for Healthcare Epidemiology of America. Pharmacotherapy 2009, 29:593607.

9. Viale P, Giannella M, Bartoletti M, Tedeschi S, Lewis R. Considerations about antimicrobial stewardship in settings with epidemic extended-Spectrum beta-lactamase-producing or Carbapenem-resistant Enterobacteriaceae. Infect Dis Ther. 2015:4:65-83.

10. Hwang H, Kim B. Impact of an infectious diseases specialist-led antimicrobial stewardship programmes on antibiotic use and antimicrobial resistance in a large Korean hospital. Sci Rep. 2018;8:14757.

11. LaRosa LA, Fishman NO, Lautenbach E, Koppel RJ, Morales KH, Linkin DR. Evaluation of antimicrobial therapy orders circumventing an antimicrobial stewardship program: investigating the strategy of "stealth dosing". Infect Control Hosp Epidemiol. 2007;28:551-6.

12. Kalil AC, Metersky ML, Klompas M, Muscedere J, Sweeney DA, Palmer LB, Napolitano LM, O'Grady NP, Bartlett JG, Carratala J, et al. Management of Adults with Hospital-acquired and Ventilator-associated Pneumonia: 2016 clinical practice guidelines by the Infectious Diseases Society of America and the American Thoracic Society. Clin Infect Dis. 2016;63:e61-e111.

13. Task Force on CAP, Philippines: Diagnosis, Empiric Management, and Prevention of Community-Acquired Pneumonia in Immunocompetent Adults 2016 Update.

14. Task Force on UTI, Philippines: Philippine Clinical Practice Guidelines on the Diagnosis and Management of Urinary Tract Infections in Adults 2013 Update.

15. Kim J, Craft DW, Katzman M. Building an antimicrobial stewardship program: cooperative roles for pharmacists, infectious diseases specialists, and clinical microbiologists. Lab Med. 2015:46:e65-71.

16. Leekha S, Terrell CL, Edson RS. General principles of antimicrobial therapy. Mayo Clin Proc. 2011;86:156-67.

17. Morency-Potvin P, Schwartz DN, Weinstein RA. Antimicrobial stewardship: how the microbiology laboratory can right the ship. Clin Microbiol Rev. 2017:30:381-407

18. Fox-Lewis S, Pol S, Miliya T, Day NPJ, Turner P, Turner C. Utilization of a clinical microbiology service at a Cambodian paediatric hospital and its impact on appropriate antimicrobial prescribing. J Antimicrob Chemother. 2018;73:509-16.

19. Masterton RG. Antibiotic de-escalation. Crit Care Clin. 2011;27:149-62.
20. Paul M, Dickstein Y, Raz-Pasteur A. Antibiotic de-escalation for bloodstream infections and pneumonia: systematic review and meta-analysis. Clinical microbiology and infection: the official publication of the European Society of Clinical Microbiology and Infectious Diseases. 2016;22:960-967.

21. Liu P, Ohl C, Johnson J, Williamson J, Beardsley J, Luther V. Frequency of empiric antibiotic de-escalation in an acute care hospital with an established antimicrobial stewardship program. BMC Infect Dis. 2016;16:751.

22. Meeker D, Linder JA, Fox CR, et al. Effect of behavioral interventions on inappropriate antibiotic prescribing among primary care practices: a randomized clinical trial. JAMA. 2016;315:562-70.

23. Antibiotic Resistance Threats in the United States. Centers for Disease Contro and Prevention; 2013. https://www.cdc.gov/drugresistance/pdf/ar-threats-2013508.pdf

24. WHO Global Strategy for Containment of Antimicrobial Resistance. World Health Organization; 2001. https://www.who.int/drugresistance/WHO Global_Strategy_English.pdf.

25. Antimicrobial Resistance Surveillance Report, Department of Health Research Institute for Tropical Medicine, Philippines. 2017. https://arsp.com. ph/wp-content/uploads/2018/07/2017_annual_report_summary.pdf.

\section{Publisher's Note}

Springer Nature remains neutral with regard to jurisdictional claims in published maps and institutional affiliations.
Ready to submit your research? Choose BMC and benefit from:

- fast, convenient online submission

- thorough peer review by experienced researchers in your field

- rapid publication on acceptance

- support for research data, including large and complex data types

- gold Open Access which fosters wider collaboration and increased citations

- maximum visibility for your research: over $100 \mathrm{M}$ website views per year

At $\mathrm{BMC}$, research is always in progress.

Learn more biomedcentral.com/submissions 